\title{
Chronic adult T-cell Leukemia in a young male after blood transfusion as a newborn
}

\author{
Magali Colucci1', Camila Cánepa², Matías Ruggieri², Carolina Berini², Nora Halperin1, \\ Francisca Rojas', Alejandra Altube1, Cecilia Cabral Lorenzo3, Alberto Deves ${ }^{3}$, \\ Olivier Hermine ${ }^{4}$, Mirna Biglione ${ }^{2}$
}

\author{
1Department of Haematology, Hospital de Clínicas "José de San Martín", Ciudad de Buenos Aires, Argentina \\ ${ }^{2}$ Department of Microbiology, Instituto de Investigaciones Biomédicas en Retrovirus y SIDA, UBA- \\ CONICET, Ciudad de Buenos Aires, Argentina \\ ${ }^{3}$ Department of Pathology, Hospital de Clínicas "José de San Martín", Ciudad de Buenos Aires, Argentina \\ ${ }^{4}$ Department of Hematology, Paris Descartes University, Paris, France
}

Received February 03, 2016; Revised June 03, 2016; Accepted June 04, 2016; Published Online June 23, 2016

\section{Case Report}

\begin{abstract}
Human T-cell Lymphotropic virus type 1 (HTLV-1) is the etiological agent of Adult T-cell Leukemia/Lymphoma (ATLL) and HTLV-1 Associated Myelopathy/Tropical Spastic Paraparesis (HTM/TSP). Areas of extremely high HTLV-1 prevalence are surrounded by areas of middle or very low prevalence. ATLL is an aggressive lymphoproliferative malignancy of peripheral $\mathrm{T}$ cells, with an incidence of less than $5 \%$ in HTLV-1-infected individuals. ATLL developed in the majority of cases in individuals who were infected with HTLV-1 by their mothers due to prolonged breastfeeding. In non-endemic areas, ATLL is usually limited to immigrants, their sexual partners and descendants from endemic regions. Very few cases of ATLL have been diagnosed in recipient patients few years after an organ transplantation or blood transfusion worldwide. Achieving an accurate and fast diagnosis of ATLL can be challenging due to the lack of professional experience, delayed consultation and difficulty in its sub-classification. We present a case of a delayed onset of a chronic ATLL in an 18-years-old male who was transfused with blood components as a premature newborn in Buenos Aires, a non-endemic city of South America.
\end{abstract}

Keywords: ATLL; Chronic, HTLV-1; Blood transfusion; Latency

\section{Introduction}

Human T-Cell Leukemia Virus Type 1 (HTLV-1) is the etiological agent of Adult T-Cell Lymphoma/Leukemia (ATLL) and HTLV-1-Associated Myelopathy/Tropical Spastic Paraparesis (HAM/TSP). HTLV-1 can be acquired by mother-to-child transmission through prolonged breastfeeding or sexual and parenteral transmission. Approximately 5-10 million individuals are infected with HTLV-1 worldwide and, among them, $1 \%-5 \%$ will develop an associated pathology. HTLV-1 infection tends to be focally endemic in well-defined geographic regions. In non-endemic areas, HTLV-1 infection is reported among immigrants, their sexual partners and descendants from endemic areas and among at-risk populations. ${ }^{1}$ ATLL is a highly aggressive neoplasm of pleomorphic activated $\mathrm{T}$ lymphocytes, which develops in the majority of cases in adulthood after a long latency since, in virtually all cases, individuals are infected by their mother due to prolonged breastfeeding. ${ }^{2}$ Worldwide very few cases of ATLL have been diagnosed in recipient patients 2 to 3 years after blood transfusion or organ transplantation.$^{3,4}$ Recently, a case of ATLL has been demonstrated after sexual transmission. ${ }^{5}$ The diagnosis criteria for ATLL includes the detection of antibodies against HTLV-1 in the peripheral blood of a patient with T-Cell Lymphoma or Leukemia and the presence of typical lymphocytes ("flower cells") circulating in peripheral blood of patients with leukemic manifestations. In case of tumors, a monoclonal insertion of HTLV-1 proviral DNA into the tumor cells is also observed. Immuno phenotypic diagnosis is also required, tumor cells are of $\mathrm{T}$ cell origin and exhibit a mature activated memory

\footnotetext{
Corresponding author: Mirna M Biglione; INBIRS, UBA-CONICET, Paraguay 2155, piso 11, C1121ABG, CABA, Argentina. 
CD4+CD25+ phenotype. ${ }^{6}$ ATLL is an heterogeneous disease clinically divided into four subtypes (acute, lymphoma, chronic and smoldering) according to leukemic manifestations in the blood, organ involvement, serum lactate dehydrogenase (LDH) levels and corrected serum calcium levels; skin lesion may be present. ${ }^{7}$ The most common form of presentation is acute, characterized by the presence of organomegaly and leukemic cells in the blood. Hypercalcaemia with or without lytic bone lesions is present in half of these patients. The chronic form is characterized by lymphocytosis, which may be stable for months or even years, skin manifestations, no organomegaly except for lymph nodes, absence of hypercalcemia and usually normal LDH levels. The chronic type progress is much slower, less aggressive, and is divided into two subtypes: the unfavorable chronic type and the favorable chronic type with no poor prognostic factors (normal values for serum lactate dehydrogenase (LDH), serum blood urea nitrogen and serum albumin). ${ }^{8}$ Patients with smoldering ATLL are usually asymptomatic or manifest skin rashes and there is no lymphocytosis. Lymphoma presents with no evidence of blood involvement but involvement of organs, lymph nodes, hypercalcemia and high LDH level. The rate of survival varies depending on the subtype ranging from 4 to 6 months for the acute type to 34 months to more than 5 years for the smoldering type. Antiviral therapy is currently the standard treatment in Europe and USA for patients with ATLL (except for the lymphoma type). ${ }^{9}$ We report the case of a young Argentine male, whose mother was HTLV-1 negative, who developed a chronic ATLL at the age of 18 after receiving a blood transfusion in the first months of life in the non-endemic capital city of Argentina.

\section{Case presentation}

An 18-year-old Argentine male attended for the first time a dermatology service of a Public Hospital in April 2015 due to a periorbital edema and cutaneous nodular lesions in the right side of the face, which were soft in consistency and not adhered to deep plans. The patient complained of painless hassle sensation in the right eye for 6 months prior to consultation and until aesthetical discomfort (Figure 1A).

The patient underwent a thorough evaluation to exclude nodular fasciitis and other differential diagnosis. Human immunodeficiency virus serology was negative and there was no evidence of an underlying immunocompromised status. No other sign or symptom were identified; however, peripheral blood examination revealed leukocytosis and 70\% of neutrophils among 20.000 white blood cells (WBCs) in blood smear. A rheumatic disease was suspected, but three months later (in July), a lymphocytosis $\left(14,000\right.$ lymphocytes/ $\mathrm{mm}^{3}$ ) was observed and the patient was derived to the haematology unit. A new examination determined abnormal $\mathrm{T}$ lymphocytes with a characteristic phenotype: $\mathrm{CD} 3+, \mathrm{CD} 4+, \mathrm{TCR} \alpha \beta+, \mathrm{TCR} \gamma \delta-, \mathrm{CD} 25+, \mathrm{CD} 10-$, CD8-, CD28+, CD5+, CD2+, (78\%), CD7-/HLA-DR-, HLA-DR+D by flow cytometry supporting a $\mathrm{T}$ cell lymphoproliferative disorder suggestive of Non-Hodgking's Lymphoma (NHL). Two months later, an eyelid cutaneous biopsy showed $\mathrm{T}$ lymphocytes infiltration with immunophenotype compatible with peripheral T-Cell Lymphoma and a bone marrow biopsy confirmed the diagnosis (Figure 2).

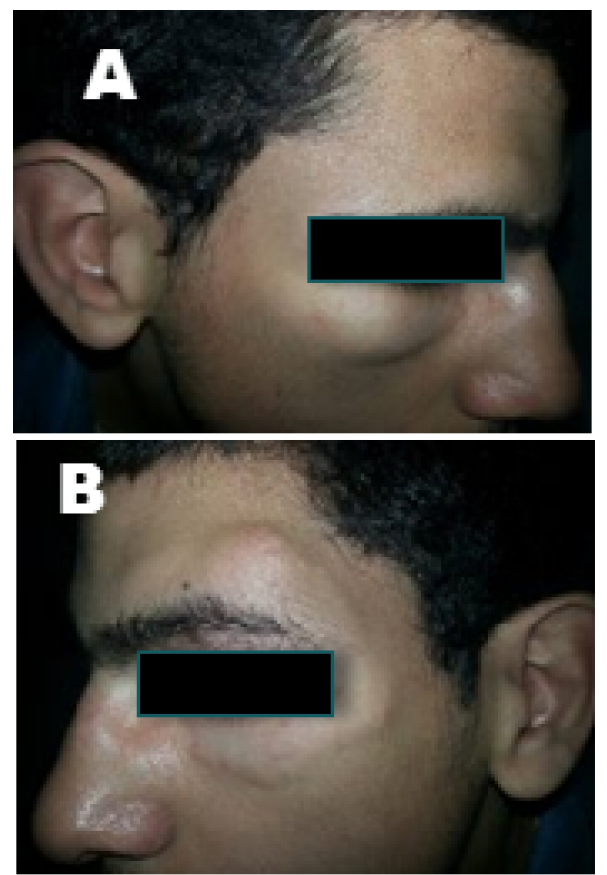

Figure 1: Photograph of the patient showing right $(\mathrm{A})$ and left (B) periorbital edema and cutaneous nodule.

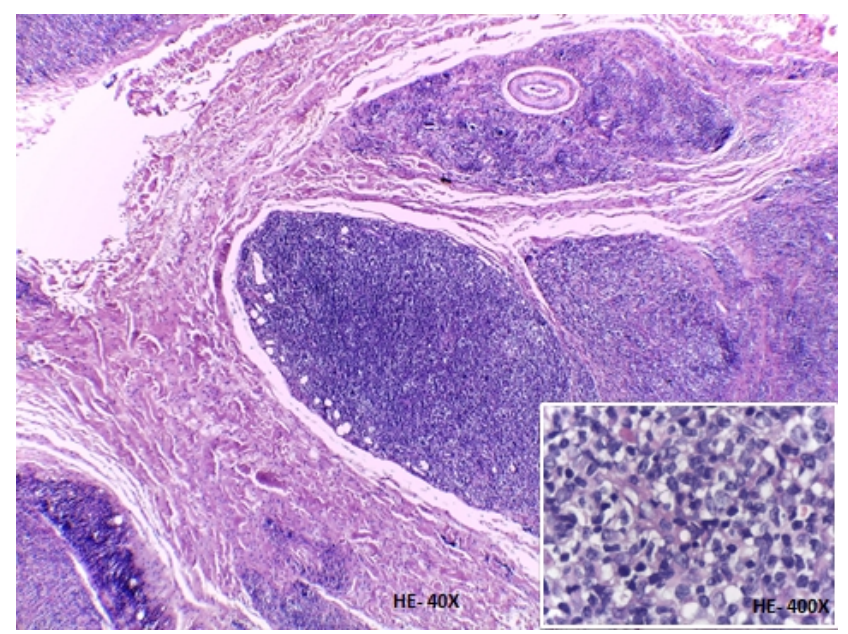

Figure 2: Histopathology of skin nodule (left forearm) showing the subepithelial tissue infiltrated by a population of uniform, predominantly small-sized T lymphocytes with folded nuclei. 


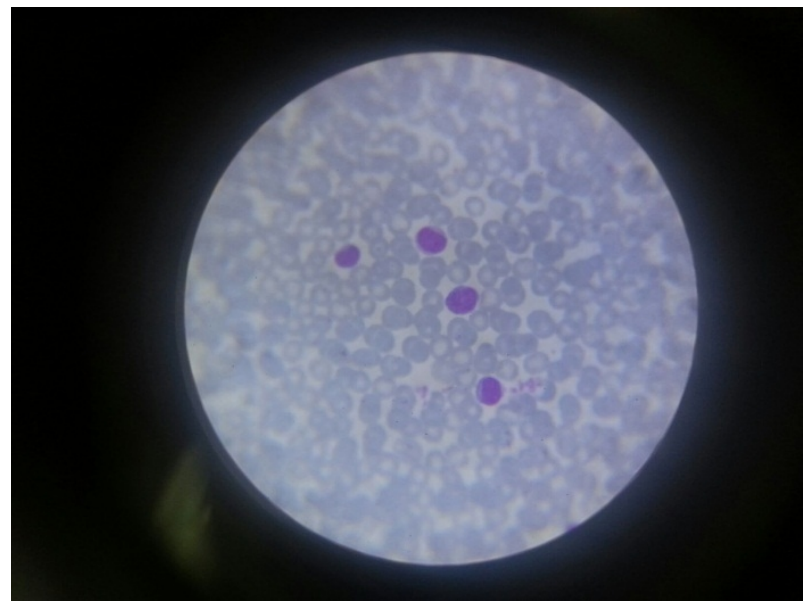

Figure 3: Blood peripheral smear showing typical abnormal medium size T-lymphocytes, with agranular and weakly basophilic cytoplasm and convoluted nucleus, named "flower cells" characteristic of ATLL (May-Grünwald Giemsa staining, 100X).

Diagnosis for HTLV-1/2 infection was performed. A screening immunoassay (Particle-agglutination assay, Serodia HTLV-1, Fujirebio Inc, Tokyo, Japan) was reactive and the result was confirmed by Western Blot (MP Diagnostics HTLV BLOT 2.4, Singapore), giving a typical complete sero-reactivity (p19, p24, p53, GD21, and MTA-1) to HTLV-1 infection. Proviral load was determined in peripheral blood mononuclear cells (PBMCs) (9,8 copies/100 cells) by quantitative real time PCR (q-PCR) and 5\% typical "flower" cells were observed in the blood smears (Figure 3 ).

The patient reported not to have ever had sexual contacts, so mother to child transmission was suspected. The mother has undergone HTLV-1/2 diagnosis and was confirmed serologically and molecularly as negative for HTLV-1/2 infection. A deeply clinical survey was performed to the patient and his mother twice, with different physicians and separately in order to reveal the transmission route. He was born and raised in Buenos Aires city, had no sexual partners, however the mother reported two blood transfusions received by the patient during his first month of life as he was premature and suffered of a serious hyaline illness. After 40 days (in September), the size of the nodule had grown and similar ones appeared at the right forearm and at the other side of the face (Figure $1 \mathrm{~B}$ ).

No systemic symptoms were present and computed-tomography scans showed no lymph nodes, organomegaly or lytic bone lesions. Absence of hypercalcaemia and laboratory parameters with no poor prognostic factors finally classified ATLL as a favorable chronic type with skin manifestations according to the Shimoyama classification. ${ }^{7,8} \mathrm{~A}$ combination of zidovudine (600 mg/day)/ pegylated IFN-alpha (90ucg/kg/week) was initiated. At the last follow-up visit (in June), lymphocytosis decreased as well as both facial nodules diminished without other abnormalities. Other factors (calcium and LDH) had normal values. The patient's written consent was obtained for this report.

\section{Discussion}

This reported case is remarkable for two reasons: firstly, the development of a favorable chronic ATLL after HTLV-1 transmission by blood transfusion, is very rarely reported. Secondly, our patient had an asymptomatic course of HTLV-1 infection and developed the disease at 18 years old exhibiting cutaneous manifestations, in opposition to the commonly early onset of postransfutional ATLL. This delayed onset after transfusion, but earlier in comparison to infection through breastfeeding might be due to reduce immune response at the time of infection in this premature newborn.

In this case, the patient's mother was negative for HTLV-1 infection and the only risk factor found was the two blood transfusions received as a newborn in 1997. Although, the detection of anti-HTLV-1/2 antibodies had been recommended in Argentina in that year by the Immunohemathology and Hemotherapy Argentine Association (AAHI), it only became mandatory 8 years later, in 2005. This case highlights the challenge in reaching an accurate and timely diagnosis of ATLL and its sub-classification in areas were the virus is not endemic. A close coordination between physicians, dermatologists and hematologists is vital in patients with this disease, as a misdiagnosis can have important impact in their life expectancy. Particularly, in these chronic forms of the disease it is interesting to notify that treatment with chemotherapy is not efficient and often is worse than the watch and wait policy In contrast, the combination of AZT and Interferon may induce a high rate of response, which may last long. ${ }^{10}$

\section{Conclusion}

This case describes the occurrence of favorable chronic ATLL with cutaneous manifestation as first symptom in an Argentine young male patient. This data confirms the hypothesis that ATLL can develop following blood transfusion, apart from breastfeeding, with a delayed onset after infection at least in premature newborns. The possibility of developing ATLL due to seropositive blood transfusion should be kept in mind considering the date of mandatory screening for HTLV-1 in blood banks and the implementation of this surveillance in each country.

\section{Conflict of interest}

The authors declare that they have no conflicts of interest. The authors alone are responsible for the content and writing of the paper. 


\section{Acknowledgement}

This research was supported by CONICET (grant: PIP112 20110100644) and BIOARS.

\section{References}

1. Gessain A, Cassar O. Epidemiological Aspects and World Distribution of HTLV-1 Infection. Front Microbiol. 2012; 3:388.

2. Hino S. Establishment of the milk-borne transmission as a key factor for the peculiar endemicity of human T-lymphotropic virus type 1 (HTLV-1): the ATL Prevention Program Nagasaki. Proc Jpn Acad Ser B Phys Biol Sci 2011; 87:152-66.

3. Glowacka I, Korn K, Potthoff SA, et al. Delayed Seroconversion and Rapid Onset of Lymphoproliferative Disease After Transmission of Human T-Cell Lymphotropic Virus Type 1 From a Multiorgan Donor. Clin infectious disease. 2013; 1417-24.

4. Pombo-de-Oliveira MS, Carvalho SM, Borducchi $\mathrm{D}$, et al. Adult T-cell leukemia/lymphoma and cluster of HTLV-I associated diseases in Brazilian settings. Leuk Lymphoma. 2001; 42(1-2):135-44.

5. Sibon D, Cassar O, Duga I, et al. Adult T-Cell Leukemia/Lymphoma in a Caucasian Patient After Sexual Transmission of Human T-Cell Lymphotropic Virus Type 1. Open Forum Infect Dis. 2015; 6;2(2):ofv032.

6. Matutes E. Adult T-cell leukaemia/lymphoma. Journal of Clinical Pathology. 2007; 60(12):1373-77.

7. Shimoyama M. Diagnostic criteria and classification of clinical subtypes of adult T-cell leukaemia-lymphoma. A report from the lymphoma study group (1984-87) British Journal of Haematology.1991; 79(3):428-37.

8. Atae Utsunomiya, Ilseung Choi, Dai Chihara et $a l$. Recent advances in the treatment of adult T-cell leukemia-lymphomas. Review Article. Cancer Sci. 2015; 106: 344-51.

9. Ishitsuka K, Tamura K. Treatment of adult T-cell leukemia/lymphoma: past, present, and future. European Journal of Haematology. 2008; 80(3):185-96.

10. Bazarbachi A, Plumelle Y, Carlos Ramos J, et al. Meta-analysis on the use of zidovudine and interferon-alfa in adult T-cell leukemia/lymphoma showing improved survival in the leukemic subtypes. J Clin Oncol. 2010;28(27):4177-83. 